Digilec 5 (2018), pp. 36-51

Fecha de recepción: 28/12/2017

Fecha de aceptación: 19/01/2019

DOI: https://doi.org/10.17979/digilec.2018.5.0.3241

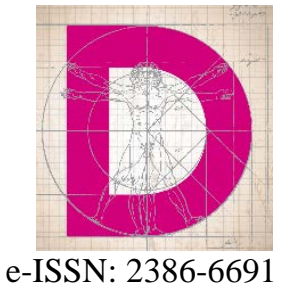

\title{
HACIA UNA DIDÁCTICA DE LA LENGUA MINORIZADA EN CONTEXTOS DE ASIMILACIÓN LINGÜÍSTICA. ALGUNAS APORTACIONES DESDE LA TEORÍA
}

\author{
TOWARDS A DIDACTICS OF THE MINORITIZED LANGUAGE IN \\ CONTEXTS OF LINGUISTIC ASSIMILATION. SOME \\ CONTRIBUTIONS FROM THEORY
}

\author{
Iris Orosia CAMPOS BANDRÉS* \\ Universidad de Zaragoza
}

\begin{abstract}
Resumen
Actualmente, alrededor de 120 lenguas europeas son denominadas como regionales o minoritarias y solo el 10\% de los idiomas del mundo están fuera de riesgo de desaparición. Uno de los principales campos para la protección de las lenguas en peligro es el educativo. Sin embargo, algunos territorios plurilingües todavía no otorgan a sus lenguas propias un estatus suficiente para garantizar su supervivencia. No obstante, en estos contextos de asimilación lingüística, donde los modelos de educación multilingüe no tienen cabida, pueden resultar relevantes algunas claves que mejoren en cierta medida la situación de la lengua minorizada en las aulas. Para desarrollarlas, se revisan los trabajos de Arnau y Mendoza y los principios de la Sociodidáctica de las Lenguas.
\end{abstract}

Palabras clave: Sociodidáctica de las Lenguas; lengua en peligro; lengua minoritaria; lengua propia; multilingüismo.

\begin{abstract}
Nowadays, in the European territories, about 120 langauges have the status of regional or minority languages and only $10 \%$ of world's languages are out of danger of disappearing. One of the main areas to protect minority languages is the educational one. Nevertheless, there are still some plurilingual states and/or territories that have not given to their regional languages the status they need in order not to disappear in the future. In the schools of these territories, where multilingual education is not developed and the language is taught as a subject, it is important to consider some didactical characteristics that can improve the situation of that regional and minoritized languages. In order to uderline these keys we have reviewed the works of Arnau (2005) and Mendoza (2005) and also the principles of the Sociodidactics of Languages.
\end{abstract}

Key Words: Sociodidactics of Languages; endangered language; minority language; regional language; multilingualism.

* Departamento de Didáctica de las Lenguas y de las Ciencias Humanas y Sociales. Facultad de Ciencias Humanas y de la Educación de la Universidad de Zaragoza (Campus de Huesca). C/ Valentín Carderera, 4, 22003, Huesca. Email: icamposb@unizar.es 


\section{INTRODUCCIÓN}

De un siglo a esta parte, el acelerado desarrollo de las tecnologías de la información y la comunicación ha derivado en un mundo globalizado en el que cada día toma más relevancia la capacidad de comunicación entre ciudadanos de diferentes territorios y culturas. En consecuencia, durante las últimas décadas se ha desarrollado un interés sin precedentes por el conocimiento de múltiples idiomas y, sobre todo, los de empleo mayoritario, dado que se presentan como herramientas casi indispensables para alcanzar el éxito académico y profesional. Este hecho ha derivado en la supremacía de una serie de lenguas-herramienta, entre las cuales destaca el inglés, considerada ya como lengua internacional de poder económico y político (Fishman, 2001; Moreno Cabrera, 2008). En síntesis, nos encontramos en un momento sin precedentes históricos ya que, en palabras de Siguán (2005: 1999), “en ningún momento de la historia había ocurrido que todos los habitantes jóvenes de un conjunto de países recibiesen una preparación sistemática para poder comunicarse en una lengua extranjera”. Sin embargo, y de forma paradójica, este creciente interés por la comunicación y el aprendizaje de lenguas de empleo mayoritario está contribuyendo y acelerando la desaparición de muchas otras de utilización minoritaria, idiomas que no son sino el reflejo de las culturas de carácter local que también se han visto paulatinamente absorbidas por una nueva cultura globalizada. Expertos e instituciones internacionales (Austin y Sallabank, 2011; Consejo de Europa, 1992, 1995, 2010; Crystal, 2000; Fishman, 1991; Gorter, Zenotz y Cenoz, 2014;Moreno Cabrera, 2000; Moseley, 2010; Nettle y Romaine, 2000; Parlamento Europeo, 1987, 1994 y 2013; Skutnabb-Kangas, Mohanty, Panda y Phillipson, 2009) han alertado a lo largo de las últimas décadas sobre el grave estado de peligro de extinción en el que se encuentra la mayor parte de los idiomas del mundo, y estiman que a lo largo del presente siglo desaparecerán aproximadamente la mitad de las 7.000 lenguas que se hablan en la actualidad $^{1}$ (Lewis, 2009).

En nuestros días solo alrededor del $10 \%$ de las lenguas del planeta se encuentra exento de riesgo de desaparición (Crystal, 2000) y un total de 1.979 cuentan con tan solo entre 1.000 y 10.000 hablantes. En lo que respecta concretamente a Europa, alrededor de 120 lenguas se establecen bajo la tipología de lengua “regional” o “minoritaria”, lo cual significa que son lenguas diferentes a la oficial del Estado y habladas de forma tradicional en un territorio por un grupo de ciudadanos inferior al resto de la población estatal (Consejo de Europa, 1992). A consecuencia de esta realidad, durante las últimas décadas las instituciones europeas han aumentado su preocupación por la conservación de las lenguas regionales o minoritarias. En este sentido, sobre todo desde las dos últimas décadas del siglo XX, instituciones como las Naciones Unidas (UNESCO, 1996), el Consejo de Europa (1992, 1995, 2003, 2010), la Comisión Europea (1996) o el

\footnotetext{
${ }^{1}$ Se desconoce el número exacto. El Ethnologue, entidad dedicada a la catalogación de las lenguas del mundo, señala un total de 7.097 lenguas vivas en nuestros días [última consulta el 11/08/16]. Sin embargo la cifra varía en función de la fuente consultada, hecho que se debe principalmente a la controversia que existe en la clasificación de algunas realidades lingüísticas como 'lenguas', 'variedades', 'dialectos' o 'hablas'.
} 
Parlamento Europeo (1981, 1987, 1991, 2013) han emitido múltiples resoluciones e informes en los que se reitera la conveniencia de proteger las lenguas minoritarias y se ofrecen recomendaciones para hacer efectiva su salvaguarda. Uno de los hitos a este respecto fue la creación en 1992 del “European Bureau for Lesser Used Languages” y la aprobación en el mismo año de la Carta Europea de las Lenguas Minoritarias o Regionales (Consejo de Europa, 1992). Sin embargo, en ocasiones, las recomendaciones de dichos expertos e instituciones no se han hecho efectivas y en algunos territorios europeos apenas se han desarrollado procesos de planificación lingüística orientados a la protección de las lenguas propias, las cuales han recibido en estos casos la denominación de lengua minorizada y han contado con un desarrollo limitado a todos los niveles, incluido el ámbito escolar. En este ámbito, la minorización lingüística se traduce en una presencia muy limitada de estas lenguas, enseñadas, en el mejor de los casos, solo como asignaturas y no como vehículos de los contenidos curriculares de otras áreas u obviadas dentro de las aulas en la peor de las situaciones.

Expuesta esta situación, en este artículo nos centraremos en las posibilidades de mejora del tratamiento escolar de las lenguas propias en estado de minorización, partiendo del marco limitador que supone su propio estado minorizado en términos de enseñanza. Para ello repasamos las propuestas disponibles en la teoría, en concreto el enriquecimiento de la lengua minoritaria de Arnau (2005) y la puesta en valor de la literatura en el aula de estas lenguas propuesta por Mendoza (2005). Finalmente, sobre todo para los casos en los que la lengua minorizada no tiene un espacio propio en las aulas, planteamos la necesidad de la formación de los futuros maestros de los territorios plurilingües desde la perspectiva de la Sociodidáctica de las Lenguas (vid. Cortier y Cavalli, 2013), como modo de garantizar una mejora de la situación de estos idiomas (sobre todo en términos de estatus y, por ende, de actitudes hacia los mismos por parte de la comunidad escolar).

\section{ENFOQUE COMUNICATIVO Y ENSEÑANZA DE LA LENGUA MINORITARIA}

Desde la perspectiva didáctica, y a nivel europeo, todo el desarrollo teórico de las últimas décadas en torno a la disciplina de la Didáctica de las Lenguas ha alcanzado su máxima representación en el Common European Framework for Languages: Learning, teaching, assessment (Consejo de Europa, 2001). Desde este marco se entiende el lenguaje humano como un constructo complejo en cuyo proceso de enseñanzaaprendizaje entran en juego diferentes factores psicológicos y pedagógicos.

A pesar de su concepción de la comunicación humana desde la complejidad, este documento aborda la labor de dividir la competencia comunicativa en componentes separados, en competencias que "interactúan de manera compleja en el desarrollo de la personalidad única de cada individuo” (Consejo de Europa, 2001: 1), con el fin de facilitar la enseñanza y el aprendizaje de la L2/L3 desde una perspectiva comunicativa. El enfoque comunicativo de la enseñanza y aprendizaje de las lenguas se centra en la acción, entendiendo a los aprendices como miembros de una sociedad donde deben desarrollar 
una serie de tareas para las cuales necesitan del dominio de múltiples competencias, no solo lingüísticas, con el fin de resolver problemas dentro de entornos diversos y circunstancias específicas. Es decir, se entiende que las personas utilizan las lenguas en contextos sociales determinados que otorgan sentido a los actos de habla y que, por ende, el aprendizaje de una lengua debe centrarse en el desarrollo de las competencias necesarias para su empleo social.

Este cambio de paradigma supone el paso de la concepción del aprendizaje de una lengua como el mero desarrollo de la competencia lingüística a un enfoque mucho más amplio en el que se entiende que en cada situación comunicativa el hablante activa una serie de competencias funcionales desarrolladas gracias a sus experiencias de comunicación previas. Estas competencias que entran en juego en cada actividad lingüística se pueden clasificar en unas que son generales (saberes, destrezas, actitudes y sistemas de regulación del aprendizaje -aprender, aprender a hacer, aprender a ser y aprender a aprender-) y otras competencias que son específicamente comunicativolingüísticas y que a su vez están vinculadas a las anteriores. Las competencias comunicativo-lingüísticas tienen principalmente tres componentes: uno lingüístico, otro sociolingüístico y un tercero pragmático.

La comprensión de la enseñanza-aprendizaje de las lenguas desde el enfoque comunicativo supone la concepción del aprendizaje de una lengua a través de su uso, dado que solo de este modo el aprendiz puede utilizar y controlar las diferentes competencias que tiene a su disposición para llevar a cabo una tarea, entendida esta como cualquier acción intencionada que el sujeto considera necesario realizar para la resolución de un problema o para el abordaje de un objetivo, en este caso comunicativo, en un contexto específico.

En coherencia con lo expuesto, desde este enfoque se entiende que para aprender una lengua el alumno aprovecha un conjunto de conocimientos y habilidades adquiridas en otros idiomas/situaciones de comunicación que contribuyen al desarrollo de unas mismas competencias básicas. Esto parece especialmente relevante para el desarrollo de la enseñanza de lenguas minoritarias, las cuales en muchos casos tienen una utilización muy restringida fuera del ámbito escolar, de modo que parece oportuno que su enseñanza se plantee en la medida de lo posible desde una perspectiva comunicativa e integrada, en la cual haya una coordinación y armonización de los objetivos, contenidos, metodologías, evaluación, etc., de la materia de lengua propia y el resto de áreas lingüísticas (trabajo que se recoge y especifica en un proyecto lingüístico de centro). Asimismo, y en coherencia con todo lo expuesto anteriormente, parece incluso más oportuna la puesta en práctica de la lengua propia como vehículo para el trabajo de cualquier tipo de contenido curricular además de los relacionados de forma específica con las habilidades comunicativo-lingüísticas, con el fin de que la lengua sea completamente funcional al utilizarse en una situación comunicativa real como es la experiencia de enseñanzaaprendizaje que se desarrolla en las aulas. En relación a esta posibilidad, tal y como señala Jaffe (2007) respecto al caso del idioma córnico o cornuallés (Cornish language), una enseñanza a través de la lengua minoritaria no solo beneficia al alumnado aportando modelos y referentes de bilingüismo sino que, además, legitima la lengua minoritaria en la comunidad; le aporta un mayor estatus. 
Estas consideraciones se han tenido en cuenta en diversos territorios plurilingües estatales, europeos e internacionales (vid. Gorter, Zenotz y Cenoz, 2014) y han supuesto la evolución de la enseñanza de la lengua propia a la enseñanza bilingüe y, en última instancia, a los modelos de enseñanza multilingüe, en los cuales se entiende la competencia comunicativa como:

una competencia compleja, compuesta, que engloba la lengua materna, la o las lenguas nacionales, las lenguas de las comunidades inmigrantes, las lenguas europeas y otras. Escapa de la idea de yuxtaposición, de adición en compartimentos estancos (Guillén 2009: 50).

Por lo tanto, en las últimas décadas, en los estados europeos en los que el marco legislativo lo permite, se ha asistido a una evolución significativa en cuanto al tratamiento escolar de las lenguas propias, pasando a ser vehículos de una parte de los contenidos curriculares dentro de modelos de educación bi- y multi- lingües. En este sentido, ya se señalaba en el I Congreso Internacional “Expolingua”, celebrado en 1988, que la meta de recuperar el uso de una lengua minoritaria, en este caso el euskera, "es inalcanzable introduciendo tres o cuatro horas del euskara a modo de asignatura de lengua segunda, y menos cuando se trata de una lengua minoritaria y minorizada” (Siguán, 1989: 84). De este modo, se apuntaba que para la recuperación de una lengua es esencial generar alumnos bilingües y que esto solo se consigue a través de medios bilingües, entre los cuales la escuela es primordial. A este respecto, se destacaba que:

no basta con que algunos profesores enseñen euskara, todos los profesores del centro tienen que conocer, o al menos entender, el euskara. Y para el profesorado que no se sienta estimulado a ello deberán existir vías de reconversión, solo factibles, naturalmente, si existe una gran voluntad y una capacidad política (Siguán, 1989:84).

Cabe señalar en este sentido que otros autores como Skutnabb-Kangas (2000) han subrayado la importancia de que todo el profesorado encargado de impartir enseñanza con alumnado hablante de una lengua minoritaria tenga, al menos, una competencia bilingüe en la lengua minoritaria y la mayoritaria.

En relación a estas consideraciones, y sobre todo en lo que respecta al tratamiento de la lengua propia dentro de la enseñanza reglada, desde el ámbito sociolingüístico catalán ya se habían puesto de manifiesto a principios de la década de 1980 las limitaciones del tratamiento de la lengua minorizada como asignatura o a través de una inmersión de tipo parcial en lo que respecta al desarrollo de la competencia lingüística en catalán del alumnado. Así, tal y como recuerda Vila:

diversos treballs_-especialmente l'obra Quatre anys de català a l'escola (Alsina et al, 1983) — deixen clar que en la situació de minorització del català, la docència de l'idioma com a asignatura o fins i tot en règim d'immersió parcial no en garanteix pas l'aprenentatge (Vila, 2012: 210).

Por su parte, Artigal (1989) apuntaba, poco después, una serie de condiciones que deben darse para desarrollar con éxito la enseñanza en una L2: 1) asegurar el estatus de la L1 del alumnado, sin que esta sea menospreciada ni a nivel social ni a nivel escolar, lo cual pasa por su tratamiento de un modo específico dentro del currículum; 2) asegurar unas actitudes positivas hacia la lengua vehicular de la escuela, haciendo que el acceso a la enseñanza en esta lengua sea de carácter voluntario y 3) presentar y tratar esta nueva 
lengua para el alumnado de un modo particular que se centre en sus intereses y facilite su comprensión.

\section{EL ENRIQUECIMIENTO DE LA LENGUA MINORIZADA}

Las citas expuestas en el cierre del apartado anterior ponen de manifiesto el hecho constatado de la escasa utilidad que tiene el tratamiento escolar de una L2/L3 como una asignatura y no como un vehículo de la enseñanza, máxime cuando se trata de una lengua minoritaria y minorizada. En consecuencia, en nuestros días resulta difícil encontrar referencias que traten la didáctica de la lengua minoritaria desde un enfoque diferente al de la perspectiva integrada a la que hemos aludido (vid. Gorter, Zenotz y Cenoz, 2014).

Aunque lo óptimo y más respaldado por parte de los psicolingüistas y pedagogos que desarrollan estudios en territorios de contacto lingüístico es aplicar a los modelos educativos una perspectiva multilingüe y multicultural, consideramos que, en lo que concierne a la enseñanza de las lenguas que no cuentan con el reconocimiento legislativo que permita este tratamiento escolar, cabe tener en cuenta una serie de características didácticas que pueden llevar a una mejora de su enseñanza desde el modelo habitual con el que cuentan (enseñanza como asignatura en un horario limitado). Para desarrollarlas vamos a revisar los trabajos de Arnau (2005) sobre enseñanza enriquecida de la lengua minoritaria y de Mendoza (2005) respecto a la importancia de la literatura en la enseñanza de las lenguas minoritarias.

El primero, considera que la lengua minoritaria en la escuela tiene dos tipos de retos a los que enfrentarse, que son: aquellos que se presentan en la enseñanza de cualquier lengua y los que "derivan de las condiciones de manifiesta inferioridad en el contexto social y en el espacio y [la] valoración que se le otorga dentro del currículum” (Arnau, 2005: 214). Por lo tanto, este autor pone de manifiesto que en el caso de la enseñanza de las lenguas minoritarias y minorizadas resulta especialmente relevante realizar actividades motivadoras, no excesivamente centradas en los aspectos formales de la lengua (gramática), orientadas a despertar el interés de los niños y vinculadas con su contexto social. Así, la enseñanza de la gramática se realiza de un modo funcional, a partir de los productos elaborados por el alumno a través de una serie de actividades significativas para ellos en las que se tenga en consideración el logro desde el punto de vista de la comunicación y el grado en el que se hayan aplicado al texto que se debe producir.

Este modo de enfocar la enseñanza de la lengua, de naturaleza comunicativa y, por ende, como hemos visto, relevante para el aprendizaje de cualquier L2 o L3, cobra valor de un modo más importante si cabe en el caso de la lengua minoritaria. Para poner en práctica este tipo de estrategias, Arnau (2005) considera que hay que pasar por un enriquecimiento de la lengua minoritaria, aportándole unos procesos de enseñanza similares e incluso mejores que los aplicados para la enseñanza de la mayoritaria y que, según su consideración, pasan por la incorporación de los siguientes principios: realizar actividades interactivo-experienciales, incorporar diversos tipos de materiales y tecnologías y extender el uso de la lengua fuera de las paredes del aula y del centro. 
En lo que respecta a las actividades de tipo interactivo-experienciales, Arnau destaca como sus principales características: la integración de la lengua en los contenidos de otras materias en lugar de entenderla como una asignatura diferente de carácter puramente metalingüístico, la propuesta de contenidos que atraigan al alumnado (basados en su contexto cotidiano, sus intereses y/o motivaciones), la interrelación de las cuatro competencias en la lengua (hablar, escuchar, leer y escribir) y el trabajo desde una interacción del alumnado dentro de procesos de cooperación y colaboración. En este sentido, plantea el trabajo por proyectos como una buena metodología para la enseñanza de la lengua minoritaria, dado que permite la integración de la lengua y los contenidos de diversas áreas. Además, esta metodología es apropiada para desarrollar dinámicas de aprendizaje cooperativo y/o colaborativo. En cuanto a los temas de este tipo de proyectos, destaca que deben alejarse de los contenidos obsoletos que se suelen tratar habitualmente en los currículums y expone como ejemplos adecuados: los temas específicos de nuestra época (los medios de comunicación, las NNTT, la publicidad, etc.); los temas ligados a los valores (los derechos humanos, la xenofobia, etc.); los temas específicos de la cultura propia (narraciones orales, poemas, canciones, fiestas y tradiciones, oficios, gastronomía, etc.) y los temas relativos a intereses específicos del alumnado (música, deportes, etc.). En su trabajo, Arnau (2005) expone algunos modelos de aplicación de este tipo de enseñanza a través de experiencias realizadas en Catalunya y el País Vasco y destaca como actividades ejemplares, que contribuyen al enriquecimiento de la enseñanza de la lengua minoritaria las siguientes:

preparar un vídeo de presentación de la clase para ser enviado a otra escuela $\left(1^{\circ}\right.$ curso), hacer un reportaje sobre los carnavales ( $2^{\circ}$ curso), elaborar una guía sobre los servicios del barrio ( $3^{\circ}$ curso), preparar un informe sobre las dietas alimentarias de los alumnos ( $4^{\circ}$ curso), preparar una guía [de la Comunidad] con diversas rutas para los turistas $\left(5^{\circ}\right.$ curso) y elaborar una guía sobre las diferentes comunidades autónomas del Estado español (6 $6^{\circ}$ curso) (Arnau, 2005: 217-218).

Desde la perspectiva de la integración de lenguas y contenidos, este autor destaca la idoneidad de que estos proyectos tengan un carácter transdisciplinar e incorporen las diferentes lenguas estudiadas en las actividades que integran el proyecto. De este modo, destaca, por ejemplo, que en un proyecto sobre los mamíferos los alumnos pueden comenzar trabajando los contenidos generales del tema en la lengua propia para después centrarse en un animal concreto en el momento de realizar una actividad y esta se puede desarrollar a través de la lengua mayoritaria o/y de la extranjera.

Tal y como señala Arnau (2005) en sus ejemplos, esta metodología ya se viene desarrollando con éxito en los contextos vascohablante y catalanoparlante desde finales de la década de 1990 y en el Canadá francófono desde la década de 1980, y destaca su utilidad para la creación de distintos tipos de situaciones comunicativas como son las entrevistas, los debates, la redacción de informes, noticias o cartas, etc. Algunas de las claves para garantizar su éxito pasan por comprender la nueva concepción que esta metodología, de naturaleza funcional-comunicativa, presupone en el rol del docente como guía del aprendizaje de los diferentes equipos de trabajo. Asimismo, el trabajo de los alumnos en pequeño grupo para realizar después una interacción con el docente es 
especialmente adecuado para fomentar el uso y reflexión sobre la lengua. Además, este enriquecimiento (comunicativo) expuesto por Arnau:

obliga a los profesores a reflexionar sobre los diferentes discursos implicados en las tareas propuestas por los diversos contenidos escolares: las 'funciones lingüísticas', (narrar, describir, persuadir, argumentar) con sus correspondientes 'componentes lingüísticos’ (verbos, adjetivos, conectores, etc.) (Arnau, 2005: 222).

Por otra parte, este autor considera que la literatura también debe trabajarse desde esta aproximación interactivo-espacial, dado que de este modo estimula a los niños a pensar, hablar y leer, además de incrementar la habilidad de escribir. En la Educación Infantil y Primaria, la representación pictórica de la lectura, el aprendizaje grupal y la representación de una obra de teatro son ejemplos de esta perspectiva de trabajo en el aula. Asimismo, resulta oportuno ofrecer a los niños una exposición a géneros literarios variados dado que de este modo "los escolares aprenden a ver y contar sus experiencias de nuevas maneras, lo que les permite también observar el uso estético y competente del lenguaje” (Arnau, 2005: 221).

En cuanto al segundo de los pilares sobre los que fundamenta su discurso Arnau (2005), la integración de diversos tipos de materiales y tecnologías, recuerda las características de los niños y jóvenes de nuestros días como nativos digitales y considera que las nuevas tecnologías de la información y la comunicación (en adelante NTIC) deben incorporarse en la medida de lo posible como un recurso importante para la enseñanza de la lengua minoritaria. En este sentido, destaca que las NTIC son un canal de comunicación que crea nuevas maneras de comprender y expresarse que han de tenerse en consideración. Además, fotografías y vídeos sobre visitas o sobre el uso de la lengua pueden estimular la conversación y la escritura, así como servir como medio para la incorporación de aspectos culturales en las actividades realizadas. Asimismo, los procesadores de texto y la Internet (blogs, foros, correo electrónico, etc.) ofrecen muchas posibilidades para estimular el uso de la lengua en sus cuatro destrezas básicas. Estas herramientas se han venido utilizando con éxito durante las últimas décadas en la enseñanza de segundas y terceras lenguas (vid. Farías, Oblinovik y Orrego, 2010), pero ofrecen posibilidades más interesantes si cabe en el caso de las lenguas minorizadas, aportando soluciones a su escaso número de hablantes y la dispersión de los mismos, tal y como muestran trabajos como el de Campos (2015) en relación a la lengua aragonesa.

Para finalizar, como tercero de los pilares que estructuran el discurso de Arnau (2005) encontramos la extensión del uso de la lengua fuera de las paredes del aula y del centro. Para ello considera esencial que se realicen actividades de creación de un paisaje lingüístico en la lengua minoritaria en el centro, el envío de comunicados a las familias en la lengua en cuestión, así como su uso por otros hablantes del centro diferentes al docente encargado de impartir su enseñanza. También considera apropiado el contacto con hablantes de fuera del centro en interacciones planificadas o proyectos determinados como, por ejemplo, la entrevista a determinados hablantes para desarrollar un proyecto específico. Asimismo, es interesante que expertos en diferentes temas (arte, deporte, ciencia, etc.), entren al aula para aportar un mayor estatus al uso de la lengua, hablando de la temática en la que son expertos. 
Como hemos desarrollado en varios puntos de nuestro trabajo, parece que el enfoque multilingüe adoptado ya en un buen número de territorios plurilingües de todo el mundo se presenta como el modelo de enseñanza más adecuado para desarrollar una didáctica de la lengua minoritaria coherente con el enfoque recomendado por muchos autores (vid. Cenoz, 2009; Gorter, Zenotz y Cenoz, 2014). No debe olvidarse que adoptar una metodología multilingüe no solo queda restringido a enseñar varias lenguas sino a promover tanto la comunicación como la comprensión del otro y de su cultura, un aprendizaje que ayude al alumno a desarrollar la competencia plurilingüe y pluricultural, entendida como:

la capacidad de utilizar las lenguas para fines comunicativos y de participar en una relación intercultural en que una persona, en cuanto agente social, domina -con distinto grado- varias lenguas y posee experiencia de varias culturas. Esto no se contempla como la superposición o yuxtaposición de competencias diferenciadas, sino como la existencia de una competencia compleja e incluso compuesta que el usuario puede utilizar (Consejo de Europa, 2001: 167).

Para finalizar, volviendo al discurso de Arnau (2005), resulta adecuado y beneficioso para todo el alumnado poder aprender la lengua minoritaria desde este tipo de enfoque, por lo que parece interesante que la filosofía multilingüe impregne a toda la escuela y, en caso de que haya alumnado que no curse la lengua minoritaria, este también tenga un espacio donde pueda desarrollar una sensibilidad hacia esa lengua. Todo ello conlleva, como decíamos, la presencia de una filosofía educativa multilingüe, es decir, la consideración del multilingüismo como uno de los rasgos identitarios del centro y que implica a toda la comunidad educativa.

Mendoza (2005) también entiende el desarrollo de la competencia comunicativa en cualquier lengua, incluidas las minoritarias, desde una perspectiva pluricultural y plurilingüe. Desde esta perspectiva, hace hincapié en que para conseguir la conexión entre la lengua y la cultura existe la necesidad de:

una sensibilización de docentes y de aprendices hacia diversas formas de comunicación mediante distintos sistemas semióticos, para que la competencia comunicativa del alumnado se construya no solo para el intercambio oral y escrito, la comprensión y la recepción lectora, sino para la total participación en la diversidad de relaciones culturales de la/s comunidad/es propia/s de la lengua meta (Mendoza, 205: 119-120).

Mendoza (2005) subraya la importancia de atender al discurso literario, dado que expone diversos usos, posibilidades expresivas, etc., así como una proyección normativa y pragmática y, además, permite interconectar la lengua con la cultura. En este sentido, este autor destaca la doble función que cumple la literatura como exponente de la lengua y como medio facilitador del desarrollo de la competencia pluricultural. En el caso de las lenguas minoritarias, la mera presencia de los materiales literarios resulta relevante al poner de manifiesto las cualidades culturales de la lengua, ayudando a romper prejuicios y consolidar el aprendizaje de facetas normativas y pragmáticas al mismo tiempo. Sin embargo, a pesar del gran potencial que presenta la literatura para el desarrollo de la competencia comunicativa, cabe señalar que ha existido una tendencia a dejar a un lado este tipo de textos para primar objetivos considerados más funcionales. Sin embargo, Mendoza (2005) destaca que: 
los textos literarios son una excelente cantera de materiales que aportan a través de la lectura un amplio, variado y enriquecedor input lingüístico y cultural (...) [y] el texto literario es soporte y reflejo de los usos lingüísticos en sus funciones básicas de interrelación en la comunicación estética (Mendoza, 2005: 131-132).

Según defiende este autor, no hay que olvidar que la literatura contiene ejemplos y discursos de múltiples tipos entre los cuales se encuentran ejemplos de lengua oral y de modalidades discursivas propias de la comunicación cotidiana y no solo selecciones "estilísticas", por lo que se adapta perfectamente a los objetivos de la perspectiva comunicativa. Además, cabe destacar que desde esta perspectiva del aprendizaje de las lenguas, se considera que no es suficiente con que el aprendiz conozca la norma gramatical de la lengua meta porque este conocimiento, limitado, no garantiza la resolución de las situaciones de interacción comunicativa con una pragmática adecuada. En este sentido, como hemos señalado, la potencialidad del texto literario es considerable dado que permite el acceso a múltiples exponentes pragmáticos. De este modo, la obra literaria, que es principalmente un elemento artístico, al aplicarse en el aula despliega las posibilidades didácticas y la producción literaria se muestra como:

- Un completo material y un recurso básico para la formación lingüística integral.

- Una amplia y variada muestra de las propuestas de usos concretos que pueden derivarse de los abundantes usos que los textos literarios recogen.

- Un tipo de discurso cuya recepción (comprensión e interpretación) vincula, integra y activa los diversos saberes que requiere su lectura (...)

- Un exponente de los potenciales usos del sistema de la lengua (...) por lo que asume el valor de exponente lingüístico con los particulares usos creativos y estéticos y se presentan, conjuntamente, las vinculaciones entre lo normativo y lo pragmático.

- Una amplia muestra de input para el aprendizaje y para la formación lingüística (Mendoza, 2005: 155).

El docente debe ser el encargado de ayudar a los alumnos a comprender los múltiples significados presentes en el texto literario, y para ello debe diseñar materiales y actividades que faciliten este proceso y, además, estimulen las respuestas emocionales de sus alumnos, brindándoles la posibilidad de expresar sus opiniones y sentimientos. En este sentido Mendoza señala que la finalidad última del trabajo con la literatura es crear la base para la discusión y el pensamiento crítico en el aula.

\section{LA SOCIODIDÁCTICA DE LAS LENGUAS COMO PUNTO DE PARTIDA}

Finalmente, cabe hacer alusión a aquellos territorios donde la minorización de la lengua propia es máxima y ello se traduce en que esta ni siquiera tiene un espacio en la escuela como materia. Como expondremos a continuación, en estos contextos la labor del profesorado, a pesar de que no conozca la lengua en cuestión, puede ser especialmente relevante para garantizar unas actitudes positivas hacia la misma entre el alumnado. Para garantizar este tipo de acciones entre los docentes consideramos oportuno el desarrollo de su formación sociolingüística desde la disciplina de la Sociodidáctica de las Lenguas. 
La corriente de la Sociodidáctica de las Lenguas nació en Francia, alrededor del ámbito investigador de Grenoble, del cual formaban parte un conjunto de especialistas en didáctica y en sociolingüística que realizaron diversos trabajos interdisciplinares (vid. Dabène, 1994) en los que se acercaron dos disciplinas separadas hasta aquel momento y centradas, respectivamente, en definir los objetos de enseñanza y formación en las lenguas y en describir los usos lingüísticos de la comunidad. A partir de entonces, sobre todo en el ámbito francófono, se han desarrollado diferentes trabajos dentro de este ámbito interdisciplinar, pero fue en 2008 cuando el término alcanzó una dimensión “oficial” en el espacio europeo (Rispail y Clerc, 2013). Así, en nuestros días, esta perspectiva didáctica es plenamente asumida desde un marco europeo, tal y como muestran los proyectos desarrollados en el seno del European Centre for Modern Languages en torno a las lenguas minoritarias desde una perspectiva plurilingüe (vid. Cortier y Cavalli, 2013). En este sentido, en el contexto europeo se han desarrollado diferentes estudios e informes desde la perspectiva sociodidáctica, centrados en el desarrollo de la educación pluricultural y plurilingüe y basados en las características lingüísticas de cada contexto educativo. Un claro ejemplo de esta filosofía es la guía para la elaboración de políticas lingüísticas educativas en Europa desde una perspectiva plurilingüe, auspiciada por el Consejo de Europa (Beacco y Byram, 2007).

Entre los preceptos que fundamentan la perspectiva de la Sociodidáctica de las Lenguas destaca:

una descentralización de las reflexiones sobre la enseñanza: del objetivo secular: ‘¿qué debe enseñarse?' [y] de las preguntas recurrentes que de allí se derivan: ‘¿quién puede enseñar?, ‘¿quién merece el nombre de maestro?’, se ha pasado a otras preguntas: ‘¿quién es el estudiante al que me dirijo?, ‘¿cómo es su vida?’, ‘¿qué sabe?’, y sobre el plano lingüístico: ‘¿cuáles son sus lenguas?’, ‘¿qué habla?’, ‘¿qué conoce?’ (Rispail y Clerc, 2013: 75).

Desde la Sociodidáctica de las Lenguas se entiende que en la actualidad es difícil hablar de la "lengua materna” del alumno, ya que ahora lo más habitual es encontrar diversos grados de plurilingüismo, con una interacción entre diversas lenguas a través de experiencias lingüísticas muy variadas, las cuales llevan al alumnado a desarrollar prácticas lingüísticas complejas, matizadas y contextuales, desde el plano individual, familiar, grupal y/o de clase/social. La perspectiva sociodidáctica de las lenguas se basa en la consideración de la enseñanza de las lenguas como una actividad social y centra sus esfuerzos en la comprensión de la realidad de los alumnos, de sus vivencias lingüísticas, de sus usos y actitudes lingüísticas, así como de los contextos de aprendizaje, para diseñar unas prácticas adecuadas, contextualizadas y significativas en la enseñanza de las lenguas desde una perspectiva pluricultural y plurilingüe, entendiendo este concepto como la capacidad o competencia de las personas de practicar diferentes lenguas en niveles o grados diversos. En síntesis, Cortier y Cavalli (2013) señalan que implica tener en cuenta la especificidad de cada contexto sociolingüístico desde su aspecto:

- micro (contacto de lenguas y/o repertorios de los alumnos en el aula y en la escuela), analizándolos desde un punto de vista sociolingüístico y teniendo en cuenta el multilingüismo, las actitudes hacia las lenguas y los repertorios lingüísticos de cada alumno y de cada grupo cultural presente en la escuela; 
lo cual, a su vez, conlleva la necesidad de completarse por un cuadro institucional y curricular a nivel:

- macro: estatutos y leyes del país y/o la región, políticas lingüísticas centradas en la educación, etc.

- meso: instituciones y culturas educativas.

El desarrollo de la Sociodidáctica de las Lenguas en el aula conlleva una serie de implicaciones entre las cuales destacan:

- La comprensión de los fenómenos que afectan a la lengua propia, como por ejemplo la diglosia, y el desarrollo de una actitud empática con las situaciones derivadas de ellos.

- La sensibilidad respecto a las variedades dialectales de la lengua y la apertura y tolerancia respecto a las actitudes del alumnado, estimulando el uso de la lengua y desarrollando un método de corrección de los errores basado en la estimulación de la comunicación y no en una actitud normativa, la cual es corriente entre los profesores monolingües en la lengua dominante.

- Asumir una perspectiva de intercomprensión, desde la cual se muestre el grado de similitud de las diferentes variedades de la lengua, así como de diferentes lenguas presentes en el marco escolar como, por ejemplo, lenguas de la misma familia, que son consideradas ‘lenguas colaterales’ o ‘lenguas puente’ respecto a la lengua minoritaria.

- Desarrollar la capacidad de seleccionar y adaptar documentos auténticos (textos, material audiovisual, etc.) para integrarlos en actividades de naturaleza comunicativa y/o cultural (hacer un periódico, un espectáculo, completar el paisaje lingüístico del centro, etc.), dentro de un espacio de cooperación y/o colaboración.

Pero quizás lo más interesante en el caso de las lenguas minorizadas es que esta perspectiva implica la sensibilización con las lenguas presentes de algún modo en la escuela (incluidas, evidentemente, las propias) por parte de toda la comunidad educativa, por lo que estas deben tenerse en consideración a pesar de que no tengan un grado suficiente de legitimación jurídica y, por ende, no cuenten con una regulación en el sistema educativo. Así, desde esta perspectiva se destaca que en este tipo de contextos también pueden surgir numerosas ocasiones para acoger, poner en valor y explotar didácticamente las variedades lingüísticas presentes en el aula, estableciendo contacto entre ellas, señalando los puntos en los que convergen y divergen, solicitando la traducción de determinados elementos al alumnado, diseñando juegos en los que estén implicadas, o simplemente reflexionando sobre la diversidad y pluralidad lingüística.

En síntesis, sea por medio de la enseñanza de la lengua, en la lengua, o contando tan solo con la posibilidad de sensibilizar al alumnado sobre la pluralidad lingüística y cultural, la aproximación sociodidáctica se caracteriza por "une articulation aux différents contextes et doit se manifester dans la capacité des enseignangts à connaître et analyser ces contextes d'un point de vue sociolinguistique et à en utiliser les ressources comme matériaux pour la clase” (Cortier y Cavalli, 2013: 17). Se trata de la puesta en práctica de una perspectiva ecológica que engloba las lenguas y repertorios lingüísticos presentes en el aula, así como en los espacios familiares y comunitarios del territorio en el que se encuentra la escuela. 
Cortier y Cavalli (2013) recogen una serie de propuestas para el aula desarrolladas por docentes de diversos contextos plurilingües en los que existen lenguas propias en estado de minorización (como el corso, el francoprovenzal o el gaélico escocés), poniendo de manifiesto las posibilidades que aporta a la lengua propia la existencia de un profesorado formado desde la perspectiva sociodidáctica. Entre ellos, un ejemplo es el proyecto "Lire la ville”, relacionado con el paisaje lingüístico de la localidad en el contexto catalanófono de Perpignan.

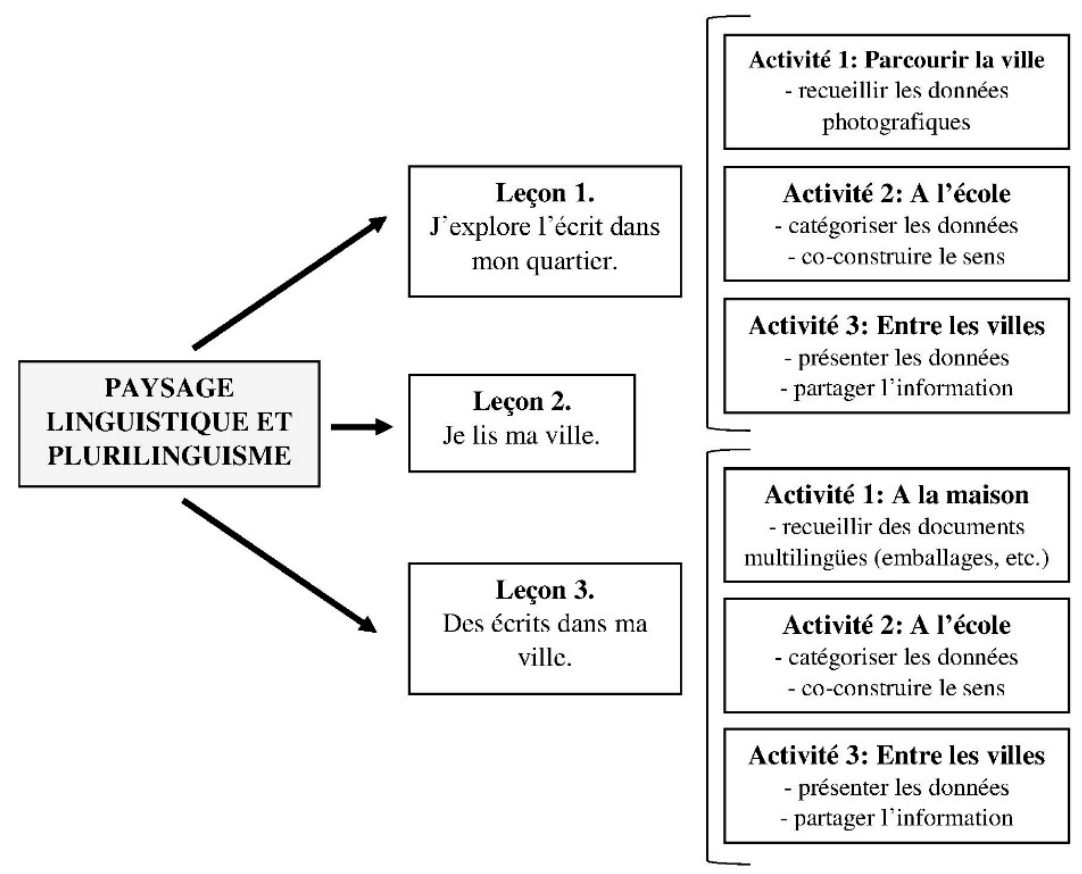

Figura 1. Síntesis del proyecto “Lire la ville”. Fuente: Cortier y Cavalli (2013).

En conclusión, parece necesario que en el ámbito de la formación de los maestros y profesores de las comunidades plurilingües del Estado, especialmente aquellas donde las lenguas propias no tienen el estatus de cooficiales, se consideren las aportaciones de la Sociodidáctica de las Lenguas con la finalidad de dar a conocer al cuerpo docente en formación la realidad plurilingüe del territorio así como las claves para su puesta en valor en su ámbito de influencia, el escolar. En este sentido, no debemos olvidar el relevante papel de la escuela en la socialización del individuo, así como el poder del profesorado como modelo y referente para el alumnado (Lasagabaster, 2003). De su sensibilidad sociolingüística dependerá en gran medida el desarrollo de la autoestima lingüística del alumnado hablante de la lengua minorizada y, en último término, el propio devenir de la misma.

\section{REFERENCIAS BIBLIOGRÁFICAS}

Arnau, J. (2005). “Nuevos retos en la enseñanza de la lengua minoritaria”. En: Llengua y lliteratura nes aules, X.A. González Riaño (Coord.), 213-229. Gijón: Academia de la Llingua Asturiana.

Artigal, J.M. (1989). La immersió a Catalunya. Consideracions psicolingüístiques i sociolingüístiques. Barcelona: Eumo Editorial. 
Austin, P.K. y Sallabank, J. (eds.) (2011). Endangered languages: beliefs and ideologies. Proceedings of the British Academy. Oxford: Oxford University Press.

Beacco, J.C. y Byram, M. (2007). Guide pour l'élaboration des politiques linguistiques éducatives en Europe - De la diversité linguistique à l'éducation plurilingue. Estrasburgo: Consejo de Europa-División de Políticas Lingüísticas.

Campos, I.O. (2015). "Las NTIC en la conservación de las lenguas minoritarias. Una investigación-acción en el ámbito educativo”. Universitas Tarraconensis. Revista de Ciències de l'Educació 1, 64-82.

Cenoz, J. (2009). Towards Multilingual Education. Basque Educational Research from an International Perspective. Clevedon: Multilingual Matters.

Comisión Europea (1996). Euromosaic. Producción y reproducción de los grupos lingüísticos minoritarios de la Unión Europea. Luxemburgo: Oficina de Publicaciones Oficiales de las Comunidades Europeas.

Consejo de Europa (1992). Carta Europea de las Lenguas Minoritarias o Regionales. http://www.coe.int/t/dg4/education/minlang/textcharter/Charter/Charter_es.pdf [Recuperado el 20/12/2017].

Consejo de Europa (1995). The Framework Convention for the Protection of National Minorities.Council of Europe, http://conventions.coe.int/Treaty/EN/Treaties/Html/157.htm [Recuperado el 20/12/2017].

Consejo de Europa (2001). Common European Framework for Languages: Learning, teaching, assessment. Estrasburgo: Council for Cultural Cooperation-Education Comittee, Language Policy Division.

Consejo de Europa (2003). Guide for the development of language education policies in Europe. From linguistic diversity to plurilingual education. Estrasburgo: Council of Europe-Language Policy Division.

Consejo de Europa (2010). Les langues minoritaires: un atout pour le développement régional, https://wcd.coe.int/ViewDoc.jsp?id=1671947\&Site=DC [Recuperado el 20/12/2017].

Cortier, C. y Cavalli, M. (eds.) (2013). Langues régionales/minoritaires dans l'éducation bi-/plurilingue. Langues d'ici, langues d'ailleurs. Viena: Ediciones del Consejo de Europa.

Crystal, D. (2000). Language death. Cambridge: Cambridge University Press.

Dabene, L. (1994). Repères sociolinguistiques pour la didactique des langues. París: Hachette.

Farías, M., Obilinovic, K., y Orrego, R. (2010). "Modelos de aprendizaje multimodal y enseñanza-aprendizaje de lenguas extranjeras”. Universitas Tarraconensis. Revista de Ciències de l'Educació, http://revistes.urv.cat/index.php/ute/article/view/631/610 [Recuperado el 20/12/2017].

Fishman, J. (1991). Reversing Language Shift: Theory and Practice of Assistance to Threatened Languages. Clevedon: Multilingual Matters.

Gorter, D., Zenotz, V. y Cenoz, J. (eds.) (2014). Minority Languages and Multilingual Education. Bridging the Local and the Global. Londres: Springer. 
Guillén, C. (2009). "La concepción y la organización del currículo de lenguas en el sistema educativo español y el plurilingüismo como competencia”. Lenguaje y Textos, 29, 47-62.

Jaffe, A. (2007). “Minority language movements”. En Bilingualism: A social approach, M. Heller (ed.), 50-70. Basingstoke/New York: Palgrave-MacMillan.

Lasagabaster, D. (2003). Trilingüismo en la enseñanza. Lleida: Milenio.

Lewis, M. P. (2009). Ethnologue: Languages of the World. Dallas: SIL International.

Mendoza, A. (2005). "Importancia de los materiales literarios en el aprendizaje de una lengua minoritaria”. En Llingua y lliteratura nes aules, X.A. González-Riaño (coord.), 119-174. Gijón: Academia de la llingua Asturiana.

Moreno Cabrera, J. C. (2000). La dignidad e igualdad de las lenguas. Crítica de la discriminación lingüística. Madrid: Alianza.

Moreno Cabrera (2008). El nacionalismo lingüístico: una ideologia destructiva. Barcelona: Península.

Moseley, Ch. (ed.) (2010). Atlas of the World's Languages in Danger. Paris: UNESCO Publishing.

Nettle, D. y Romaine, S. (2000). Vanishing voices: the extinction of the world's languages. New York: Oxford University Press.

Parlamento Europeo (1987). Resolution of the European Parliament of 30 October 1987 on the languages and cultures of regional and ethnic minorities in the European Community

(Kuijpers

Resolution), https://ospcom.files.wordpress.com/2011/10/lc4.pdf [Recuperado el 20/12/2017].

Parlamento Europeo (1994). Resolutionon linguistic and cultural minorities in the EU, http://com482.altervista.org/documents/legjislazion/killilea_en.pdf [Recuperado el 20/12/2017].

Parlamento Europeo (2013). Propuesta de resolución del Parlamento Europeo sobre las lenguas europeas amenazadas de desaparición y la diversidad lingüística en la Unión Europea, http://issuu.com/asociacionfaceira/docs/informe-alfonsi-defcas [Recuperado el 20/12/2017].

Rispail, M. y Clerc, S. (2013), "El enfoque sociodidáctico: informe de etapa y perspectivas”. En Enseñar (lenguas) en contextos multilingües, J. Dolz e I. Idiazábal (eds.), 72-87. Servicio Editorial de la Universidad del País Vasco-Euskal Herriko Unibertsitatea.

Siguán, M. (coord.) (1989). El bilingüismo en la Europa del año 2000. Madrid: Ministerio de Educación y Ciencia.

Siguán, M. (2005). La Europa de las lenguas. Madrid: Alianza.

Skutnabb-Kangas, T. (2000). Linguistic Genocide in Education - Or Worldwide Diversity and Human Rights? Mahwah y Londres: Lawrence Erlbaum Associates.

Skutnabb-Kangas, T., Phillipson, R., Mohanty, A.K. y Panda, M. (2009). Social justice through multilingual education. Bristol: Multilingual Matters.

UNESCO (1996). Universal declaration on linguistic rights, http://www.unesco.org/cpp/uk/declarations/linguistic.pdf [Recuperado el 20/12/2017]. 
Vila, F. X. (2012). “Algunes bases per a la recerca sociolingüística en sentit ampli”. En Posar-hi la base. Usos i aprenentatges lingüístics en el domini català, F.X. Vila (ed.), 11-24. Barcelona: Institut d'Estudis Catalans. 